\title{
Measurement method for construction of the radio environment maps supporting cognitive radios
}

Krzysztof Zubel, Janusz Romanik, Edward Golan, Kamil Wilgucki

Krzysztof Zubel, Janusz Romanik, Edward Golan, Kamil Wilgucki, "Measurement method for construction of the radio environment maps supporting cognitive radios," Proc. SPIE 11442, Radioelectronic Systems Conference 2019, 114421K (11 February 2020); doi: 10.1117/12.2565224

SPIE. Event: Radioelectronic Systems Conference 2019, 2019, Jachranka, Poland 


\title{
Measurement method for construction of the radio environment maps supporting cognitive radios
}

\author{
Krzysztof Zubel, Janusz Romanik*, Edward Golan, Kamil Wilgucki \\ Military Communication Institute, Radiocommunication Department, Warszawska 22A, \\ 05-130 Zegrze Poludniowe, Poland
}

\begin{abstract}
In the paper we discuss the issue of creation of Radio Environment Maps (REM). In the literature REMs are considered as an enabler for the spectral awareness and support cognitive radios and frequency management systems. We present the functional architecture of the system and describe the role of main components. Next, we present the problem of the map creation. We describe the most suitable interpolation techniques and analyze their complexity. Some interpolation techniques require high computational power and consequently created maps better reflect the signal level. Next, we focus on the problem of measurements of the signal level in sensor networks. In the literature on the topic the sensor network usually is composed of many of sensors deployed within the area of a few square kilometers. Density and regularity of sensors arrangement impacts the quality of maps. On the other hand, the number of sensors used for tests conducted in a real environment usually is significantly limited. We discuss the problem how to model the sensor network and we describe the method which we used to measure the signal level in the sensor network operating in VHF/UHF bands in scenarios with one transmitter and two simultaneous transmitters. The received signal level measured by sensors was used as an input data for the interpolation and then maps were created for the Zegrze area. The quality of maps was assessed in terms of the correctness of the signal level estimation and transmitter localization.
\end{abstract}

Keywords: Radio Environment Maps, REM, cognitive radio, spectrum management

\section{INTRODUCTION}

Coordination of NATO's joint coalition operations is a complex issue and requires resolving of the many problems. One of the important areas is the electromagnetic space, which requires advanced and effective tools to ensure proper coordination of activities at the level of allied operations. Existing spectrum management solutions are inefficient in a modern, increasingly dynamic system environment. Until now, frequency allocation is a priori and quasi-static, while spectrum control in the frequency ranges used by military systems is limited.

Currently used radio spectrum management tools require a lot of time and effort to plan allied operations. They are not sufficient support for spectrum management staff. Moreover, they do not provide a full operational picture of the spectral situation and it is very difficult to make a significant improvement in the electromagnetic situation based on them, e.g. due to the lack of current information about interferences. Being aware of the problems mentioned above, a working group was set up supported by 8 countries (France, Great Britain, USA, Belgium, Estonia, Germany, Turkey, Poland) and other entities, in particular NATO Joint Electronic Warfare Core Staff (JEWCS), which is preparing analyzes and demonstrations in the scope of development capabilities of the radio environment management systems. One of the basic tasks of the NATO IST-146 RTG-069 working group was to define the system architecture as part of the preparation of the concept of Radio Environment Maps (REM). This program is to improve situational awareness of spectrum use and ensure uninterrupted operation of radio systems in allied operations, based on interoperable REM databases.

According to the NATO IST RTG-050 plan, REM is also needed to make a significant step towards a coordinated spectrum management system in NATO [1,2].

*j.romanik@wil.waw.pl; phone + 48261 885-645; fax + 48261 885-589; www.wil.waw.pl

Radioelectronic Systems Conference 2019, edited by Piotr Kaniewski, Jan Matuszewski, Proc. of SPIE Vol. 11442, 114421K · C 2020 SPIE · CCC code: 0277-786X/20/\$21 · doi: 10.1117/12.2565224 
Chapter 2 presents the functional architecture of REM and discusses the essential elements of the radio spectrum management support system, dedicated to military applications, using radio environment maps, and discusses map creation techniques taking into account the degree of computational complexity and the quality of maps obtained. Comparison of maps obtained for different interpolation techniques allows to indicate the most promising techniques for creating radio environment maps.

Greater computational complexity necessitates the use of more efficient computers, thanks to which the created maps more accurately represent the level of radio signal at each point of the map. However, in small-scale tactical operations, the computing power of devices can be significantly reduced, and therefore other mapping techniques may be preferred.

Chapter 3 reviews the literature on modelling of sensor networks and measurement methodologies to obtain input data for creating maps by interpolation. The literature on the subject usually describes networks with several dozen or more sensors distributed over an area of several square kilometers. A large number of sensors and uniformity of distribution of the sensor network significantly affects the accuracy of mapping the level of the measured signal. In practice, among others as part of this research, the number of sensors is usually significantly limited.

The main problem is how to model the sensor network and how to make measurements. Chapter 4 discusses the method that was used to conduct tests in real conditions in the UHF and VHF band in a system with one and two transmitters working simultaneously. Measurements were made to check the impact of the number of sensors and way of arrangement on the quality of the radio environment maps. The measurement results were used as input to the interpolation process, resulting in maps of the radio environment for the Zegrze area. The maps were evaluated for mapping accuracy of the received signal level and transmitter location. Comparison of maps obtained for various interpolation techniques has allowed to indicate the most promising techniques for creating radio environment maps. The results of these works were presented at several conferences [3, 4, 5] and included in publications [6,7].

This article also shows the additional functionality of REM maps consisting in determining the location of two transmitters operating simultaneously on the same frequency.

\section{RADIO ENVIRONMENT MAPS}

\subsection{REM Concept}

The essence of REM functionality is the ability to dynamically construct radio environment maps for all desired locations and frequencies. It is assumed that REM gathers the results of spectrum measurements from dedicated sensors or radio stations with elements of cognition. Measurement data from sensors can be sent periodically or on request. Since it is not possible to place sensors in every desired location, one of the assumed functionalities of REM is to estimate the state of the spectrum in places where there are no such sensors. The literature describes several methods for constructing REM maps that are based on spatial statistics or based on the location of emission sources.

Figure 1 shows the simplified REM functional architecture [4] consisting of the following modules: Measurement Capable Devices; REM data acquisition module enabling preparation of data for further processing; REM database service module enabling writing and reading data from the REM database; REM databases; REM manager module providing database access from the graphical user interface.

Measuring devices obtain information about the current situation in the electromagnetic environment. The data acquisition module is responsible for communication with measuring devices. It sends control instructions to them, collects measurement reports, as well as transfers the collected data to the REM database service module. The database stores information about the state of the radio environment. The Manager module is responsible for maintaining the REM database. Decisions regarding the measurements taken are made in this module. It defines which measurements are to be made, which devices are to be carried out and when it is to be done. 


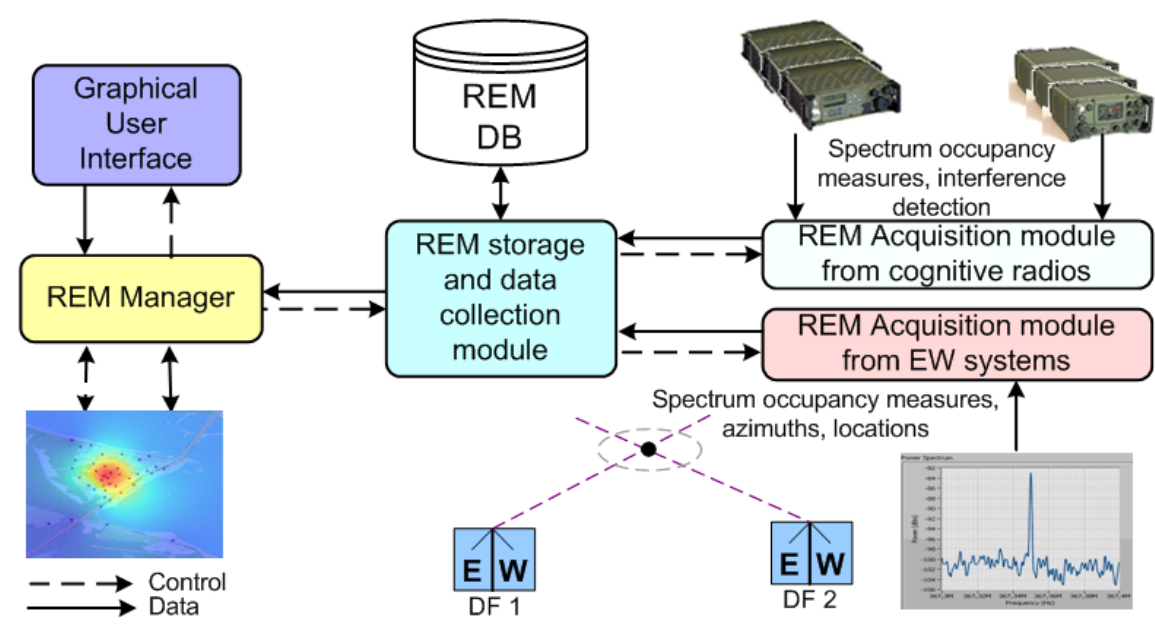

Figure 1. REM architecture [4].

The role of sensors can be performed by spectrum analyzers, radio receivers, cognitive radio stations and electronic warfare systems. Ultimately, it is assumed that the operation of sensors in term of operation mode (continuous or on demand measurement), scanned subranges and frequencies as well as the measured characteristics (e.g. the level of the received signal) will be controlled by REM Manager.

Figure 2 shows an example of REM Manager's collaboration with a dedicated sensor based on National Instruments spectrum analyzer.

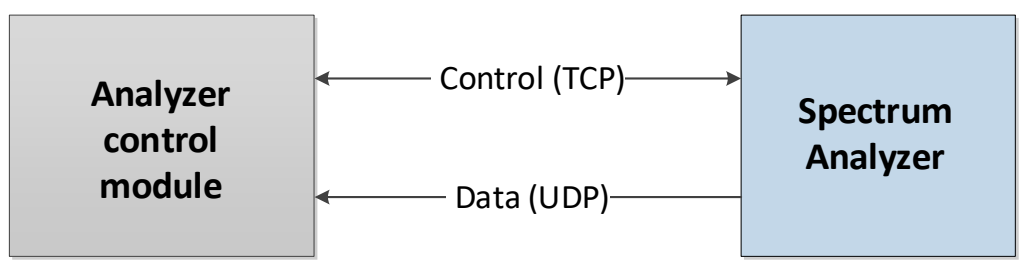

Figure 2. Communication between the Spectrum Monitor application and the Spectrum Analyzer.

The Ethernet link is used for communication between the Spectrum Monitor application located in the analyzer control module and the Spectrum Analyzer. The TCP protocol is used to send control commands, while the UDP protocol is used to send measurement results. Control commands are created in accordance with the SCPI standard. Data frames received from the UDP packet stream have the following fields:

1. "MagicNumber" - frame flag.

2. Sequence number - the number of the transmitted data frame.

3. Time stamp - contains the time the frame was created.

4. $\quad$ Reference level - set reference signal level.

5. Detection level - the minimum level above which detected signals are sent to the recipient.

6. Data length - the number of amplitudes transmitted in the data frame.

7. Step - the step of scanning the spectrum in the analyzer.

8. List of amplitudes for the scanned spectrum.

\subsection{Methods for creating REM}

The literature on the topic describes three main categories of techniques for creating radio environment map, namely: direct, indirect and hybrid $[8,9]$. Direct methods, also called methods based on spatial statistics, use interpolation of 
measured data. Indirect methods, also known as transmitter location-based methods, use transmitter location information and a radio wave propagation model to obtain a radio signal level estimate. Hybrid methods combine both methods.

Methods based on spatial statistics use measurement data obtained at specific sensor locations. This is due to the fact that placing sensors in all required places is impractical or simply impossible. Therefore, to get data in all areas, different interpolation techniques are used, treating the measurement data as input. The general assumption of the interpolation process is that sensors closer to the desired location have a greater impact on the interpolation result than sensors located at a greater distance.

In the literature on the subject $[8,9]$, the following interpolation techniques are described as the most promising: NN method (Nearest Neighbor), IDW method (Inverse Distance Vector), Kriging method and hybrid method.

The NN method is also known in the literature as proximal interpolation or point sampling. This is one of the simplest interpolation methods and thus the least accurate. The NN method uses polygons known in the literature under the name Thiessen or Voronoi polygons, which polygons are defined by demarcation lines determined at an equal distance from the points where the measurements were made. A characteristic feature of these polygons is that their boundaries are exactly halfway between adjacent measuring points. Another property of the NN method is the phenomenon of a significant difference in values represented by adjacent polygons, visible on REM maps as a sharp change in signal level.

The interpolation of the unknown value of the $P_{l}$ signal at a point with coordinates $\left(x_{l}, y_{l}\right)$ consists in assigning to this point the value of the signal measured at the nearest measuring point $P_{0}$ in the place $\left(x_{0}, y_{0}\right)$. According to the above rule, all points within a given polygon will have the same value. The advantage of this method is the low complexity of implementation and calculations, while the disadvantage is the step change in the signal value at the polygon border.

The IDW method is based on the assumption that the estimated value (e.g. level of received $P_{l}$ signal) at point $\left(x_{l}, y_{l}\right)$ is more dependent on the signal values obtained for closer measurement points. The results obtained for distant points have a much smaller impact.

The IDW method uses weighting factors $w_{i}$, which are inversely proportional to the distance between a given point $\left(x_{1}, y_{1}\right)$ and the measuring point $\left(x_{i}, y_{i}\right)$ and raised to the power of $p$. The power of $p$ determines how the weighting coefficients change with distance. If $p$ takes large values, then the closer points have bigger influence on the interpolation result. If, on the other hand, $p$ is zero, then regardless of the distance, the coefficient $w_{i}$ does not change. The following notation was adopted later in the article: IDW pX - means the IDW method with an exponent equal to X.

Kriging belongs to geostatistical interpolation methods. This means that it works on stochastic principles, i.e. the random variability of the interpolated feature is taken into account. Interpolation is based on the principle of finding a statistical relationship between the values corresponding to known (measuring) points, and the estimated value of interpolated point. Kriging uses the relationship between the distance of points and the degree of their dependence in terms of the level of received signal. Usually this relationship is presented on the charts called semi-variograms [4].

\section{LITERATURE OVERVIEW RELATED TO SENSOR NETWORK MODELING}

\subsection{Sensors placement in simulation models}

An ideal model for the distribution of measuring sensors within a given area is a model consisting of placing the sensors in a grid manner, which enables to cover regularly the entire area for which the REM map is being developed. The distance between the sensors is selected depending on the morphology of the terrain and the propagation characteristics of radio waves. This type of model is most often used in simulation studies in which REM map creation is only an intermediate step in the verification of other mechanisms, e.g. regarding the comparison of REM map interpolation techniques or radio emission source location methods.

The article [8] presents a simulation model where main purpose was to compare various interpolation methods enabling the creation of REM maps. The characteristic of radio wave propagation was assumed in accordance with the expanded Friis model on radio wave propagation in free space, taking into account the impact of terrain obstacles such as hills, trees and buildings. This model in the literature on the topic appears under the name "log-normal shadowing". For the simulation process an area of 1000 meters by 1000 meters was divided into squares with a side length of 250 meters. A measuring sensor was placed in the center of each square, thus creating a regular grid of 16 sensors. The exponent of the power in the formula describing the path losses was assumed to be equal to 3.5. The standard deviation in the Gaussian random distribution was assumed to be equal to $8 \mathrm{~dB}$. The radio signal source transmitting at approximately 
$-20 \mathrm{dBm}$ was located in the lower right quadrant of the simulation area. The simulation results revealed differences in the quality of the resulting REM maps obtained by means of IDW interpolation with power exponent 1 and 2 , as well as Kriging interpolation, with the advantage in favor of the latter technique.

The article [10] presents a simulation where the main purpose was to detect and determine the location of several radio emission sources using the Hidden Markov Model. The simulation assumed: the characteristics of radio wave propagation described by the Rayleigh channel with fading, the model of additive white Gaussian noise (AWGN) at an average power level of $-100 \mathrm{dBm}$, the frequency of radio sources operating at frequency of $900 \mathrm{MHz}$ and the power transmitted by sources of $30 \mathrm{dBm}$. The exponent of power in the formula describing the path losses was assumed to be equal to 4. For the radio environment described in this way, the sensor network model consisted of nine sensors arranged in a grid system at a distance of 1000 meters between sensors. The measuring area was 2000 meters by 2000 meters. Two radio emission sources were randomly placed inside this area. REM maps developed for such simulation supported by filtration using the Hidden Markov Model allowed to detect and determine the location of both radio emission sources with high precision.

\subsection{Crowdsourcing}

Another model for obtaining measurement data for the purposes of creating REM maps is the model known in literature as "crowdsourcing". This model is based on the collection of measurement data from a relatively large number of usually random sensors, mostly private smartphones, located in the area for which the REM map is being created. Currently, it is mainly used in simulation tests. However, it is more and more often considered as a practical solution, in particular with regard to $5 \mathrm{G}$ technology.

The paper [11] presents the results of simulation tests for 5G technology in the field of so-called context-aware resource allocation. These tests consisted in determining the level of the electromagnetic field originating from a base station at each point of the macro-cell. The base station was located in the center of a 190 by 190 meters macro cell. In this area 200 sensors were randomly placed. Next, 20 sensors were selected to form clusters for the purpose of interpolation of the radio signal level at each point of the macro-cell. In this way, REM map was created for the entire macro-cell area. Since all sensors were battery-powered, the aim of the simulation was to optimize the lifetime of the sensor network based on the intensity of use of the sensors involved in the measurements. The authors of the article proposed their own algorithm for selecting sensors for the cluster. The simulation model used radio wave propagation characteristic consistent with the "log-normal shadowing" model. The exponent of the power in the formula describing the path loss was assumed to be equal to 3. The standard deviation in the Gaussian random distribution was assumed to be equal to $6 \mathrm{~dB}$. The base station was broadcasting at $24 \mathrm{dBm}$. The resulting REM map obtained using this method was delivered to the $5 \mathrm{G}$ base station as a context that allows selection of the operating parameters of this station for communication with end devices located anywhere in the macro-cell.

An important issue regarding "crowdsourcing" is the reliability of data obtained from a large number of random sensors. This topic was taken up by the authors in paper [12] presenting a proposal for a solution under the name SecREM, aimed at increasing security understood as increasing the quality of REM maps created on a basis of data obtained with the use of the "crowdsourcing" method. In the presented work the authors assumed that in a group of 100 random measurement sources there may be at least 20 sources providing false measurement data. The reasons for data falsification can be both accidental, e.g. partial damage to the measuring device, or deliberate misinforming of the radio spectrum manager. For the basic model of the attack it was assumed that measuring sources falsifying data would overstate the readings of the signal level by $20 \mathrm{~dB}$. Further research work was done with the aim to checked the effect of higher overstatements of the radio signal levels. In the proposed SecREM solution the authors assumed that the spectrum manager has a small number (e.g. 10) of reliable and trusted sensors (core of the sensor network) that deliver measurement results to create REM maps for a given area. Then the spectrum manager extends the set of another reliable sensors with another mobile sensor detecting the signal at the level comparable to the one assumed by REM map created with the use of preliminary set of sensors. After that the REM map is updated and assessment of a subsequent mobile sensors is performed. This process is continued until the assumed boundary condition, e.g. the total number of measuring sensors required to interpolate or exceeded by the best of the other sensors of the assumed difference between the signal measured by this sensor and the value estimated by the REM map. In the above studies the authors used results of the real field measurements collected as part of the CRAWDAD project at the University of Colorado Boulder campus [13]. The measurements were carried out for the local WiMax network with the use of a portable spectrum analyzer. 


\subsection{Mobile sensor}

In some cases it is necessary to obtain real measurement data for a large area. Therefore, in addition to the crowdsourcing model, a model with the use of a mobile measurement sensor moving between pre-determined measurement points is used.

The paper [14] presents a model for obtaining measurement data at the University of Colorado (USA) with the use of a mobile sensor. The measurements were carried out to determine the effectiveness of covering the university area with the WiMax radio network operating at $2.5 \mathrm{GHz}$. For the purposes of the research all measuring points were determined in nodes of the network built of equilateral triangles with a side length of 100 meters. The area of measurement was approximately 1500 meters by 1100 meters. The base station of the WiMax network located in the middle of the lower part of the research area was transmitting at $40 \mathrm{dBm}$. Due to traffic restrictions within the university center, the authors of the research built the measuring apparatus on a two-wheeled cart, which could be driven manually or could be attached to a bicycle. The main element of the measuring apparatus was the Anritsu MS2721B portable spectrum analyzer controlled remotely via the Ethernet interface using the Virtual Instrument Software Architecture (VISA) software from National Instruments (NI). The software installed on a small laptop with the Ubuntu operating system allowed the creation of measurement scripts. A handheld Garmin GPS60 receiver was used to determine the position of the measuring trolley. The measuring antenna was mounted on a plastic support at a height of 2 meters above the ground. In a situation where a given measuring point from a previously developed grid was unavailable, the measurement was made at the closest distance from that point. Three measurements were made at each point, which were then averaged to reduce the impact of transmission channel variability caused by short-term fading. The spectrum analyzer saved the measurement results in its own (proprietary) text file format, which after returning to the laboratory was converted to a form that allowed further processing of the measurement data. The authors presented several new methods and extensions to the basic geostatistical theory, which they believe are necessary in the analysis of radio networks. For the determining of the radio network coverage for the campus terrain the method based on REM was shown to be superior to the classic method using the path loss characteristics.

In the article [15], the authors presented a method of measuring radio emissions from DVB-T digital terrestrial transmitters. The measurements were carried out in the center of Poznan (Poland) with the use of a mobile sensor built-in on a passenger car travelling along a fixed route through the city center. The measurements were carried out in typical everyday traffic conditions. Measurement samples were collected at constant intervals, while the speed of the measurement vehicle was dependent on the indications of traffic lights at each intersection. Therefore, the number of measurement samples per route points was different. The length of the measuring route was $8 \mathrm{~km}$ and ran through diversified areas from housing estates, through compact and low buildings of the Old Town Square, to recreational areas located between the Warta River and Malta Lake. The measuring apparatus set consisted of: Rohde \& Schwarz FSL6 spectrum analyzer powered from the car cigarette lighter socket, wideband omnidirectional rod antenna type AOR DA753, laptop connected to the spectrum analyzer by the Ethernet. On the laptop there was installed Matlab environment running the specialized Instrumental Control Toolbox software. In addition, on the roof of the vehicle there was a GPS receiver connected to the laptop by USB cable. The GPS receiver provided accurate information about the geographical coordinates of the point where the spectrum analyzer made measurements. The measurements were carried out for four frequencies broadcasted from two different sources. TV signal with frequency of $594 \mathrm{MHz}$ was broadcasted from a transmitter located $4.5 \mathrm{~km}$ north of the city center, while TV signals with frequencies: $490 \mathrm{MHz}, 522 \mathrm{MHz}$ and $618 \mathrm{MHz}$ were broadcasted from a transmitter located $30 \mathrm{~km}$ south of the city center. The measurements confirmed that local REM maps enable to detect the so-called TV White Spaces and thus to reuse the frequencies, e.g. by cognitive radios.

\section{OWN MEASUREMENT METHODOLOGY FOR THE RADIO ENVIRONMENT MAP}

The following chapter presents the model of the installation and measurement methodology proposed and used by the authors of the article to collect input data for the purposes of creating of radio environment maps. The research system was composed of own source of radio transmitter working with less power than DVB-T transmitters, which are quite often used as a source of radio signal in this type of research, and the mobile sensor node. The power transmitted by the source was similar to the power transmitted by real cognitive radio stations (about $10 \mathrm{~W}$ ). Practical tests were carried out in real field conditions for wide frequency range with own signal source. There were 48 measurement frequencies in the VHF and UHF frequency bands. 
The transmitting part of the measurement set for the VHF / UHF bands consisted of (Figure 3):

- $\quad$ NI PXI-5671 signal generator,

- $\quad$ ZHL-100W-GANX + power amplifier (VHF range),

- $\quad$ ZHL-16W-43-S + power amplifier (UHF range),

- $\quad 3088 \mathrm{VM}$ transmitting antenna (VHF range),

- $\quad$ RF-3187-AT320 transmitting antenna (UHF range),

- $\quad$ Trimble Thunderbolt E GPS receiver.

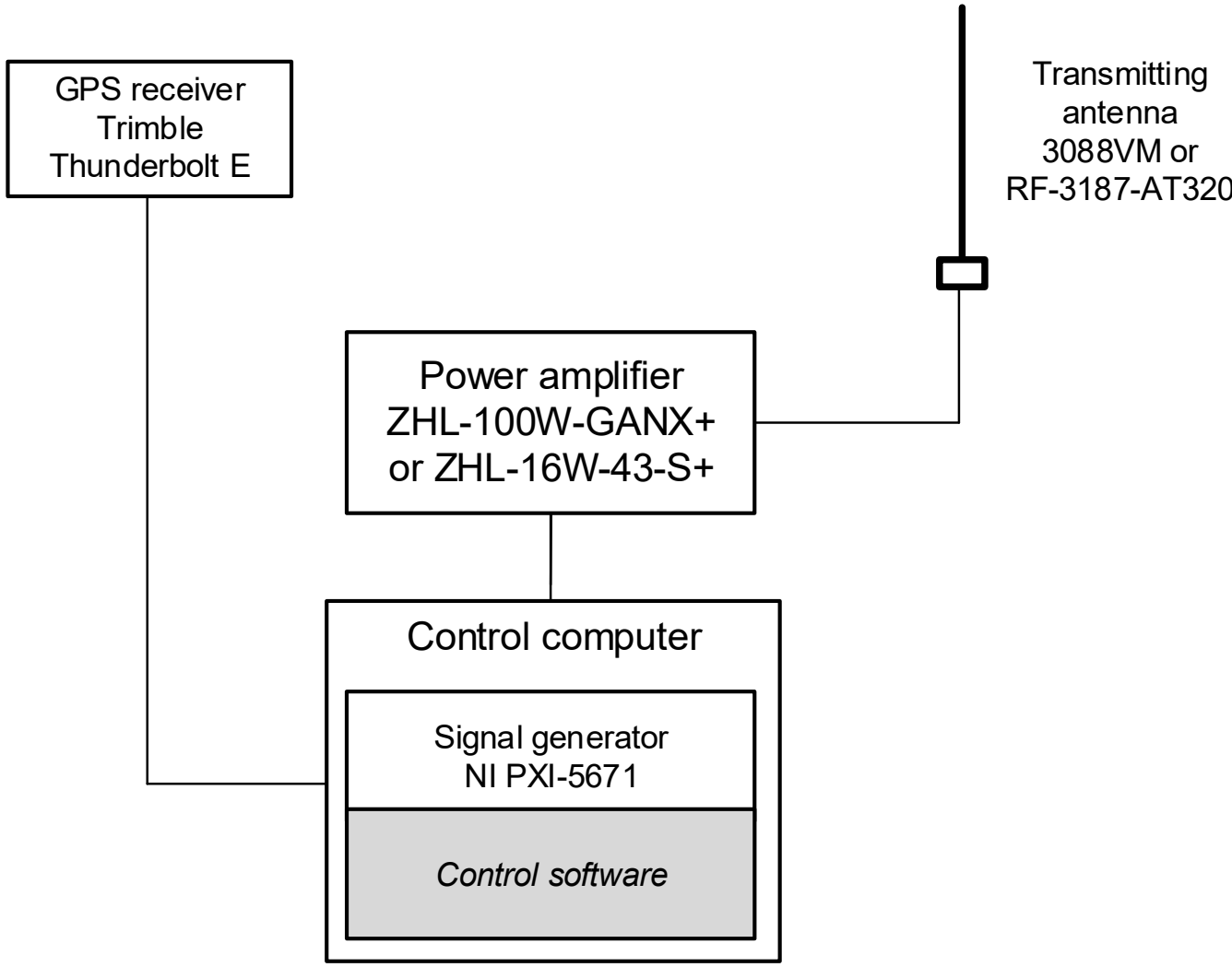

Figure 3. The transmitting part of the research kit for the VHF / UHF range.

The signal source (transmitting antenna) was placed at a height of $8 \mathrm{~m}$, which is much lower than the height of TV transmitters. It caused that there was a clearly noticeable effect of varied land cover on which the measurements were carried out on the propagation of the radio signal.

The 3088VM antenna is a typical antenna used by Polish Armed Forces designed to operate in the $30 \div 90 \mathrm{MHz}$ frequency range. The RF-3187-AT320 antenna is a three-band antenna with separate inputs for the following frequency ranges: $30 \div 100 \mathrm{MHz}, 100 \div 500 \mathrm{MHz}, 500 \div 2000 \mathrm{MHz}$. In the conducted research there were used two upper frequency ranges of this antenna.

The ZHL-100W-GAN+ power amplifier is designed to operate in the $30 \div 500 \mathrm{MHz}$ frequency range, and the ZHL$16 \mathrm{~W}-43-\mathrm{S}+$ amplifier in the $800 \div 4000 \mathrm{MHz}$ frequency range.

The NI PXI-5671 signal generator is a vector signal generator that generates a vector signal (with variable phase and amplitude) based on two streams of digital signals, e.g. from the outputs of analog to digital converters. Such an instrument is based on an IQ modulator fed by digital streams modulating phase and amplitude of the baseband reference signal and digital carrier. 
The vector signal generator is the basic device for testing modern radio communication systems and creating new studies on data modulation and coding. It allows the creation of quadrature modulated signals, i.e. those that are used in all modern radio communication standards.

Basic parameters of the NI PXI-5671 signal generator:

- $\quad$ frequency range from $250 \mathrm{kHz}$ to $2.7 \mathrm{GHz}$,

- $\quad$ built-in $32 \mathrm{MB}$ memory,

- $20 \mathrm{MHz}$ band,

- $\quad$ output power from $-145 \mathrm{dBm}$ to $+10 \mathrm{dBm}$,

- $10 \mathrm{MHz}$ internal reference signal with \pm 20 ppm stability.

The Trimble Thunderbolt E GPS receiver fulfilled the following functions:

- $\quad$ synchronization of frequency changes of the NI PXI-5671 signal generator,

- $\quad$ receive TSIP messages 0x8FAB Primary Timing Packet.

The LabVIEW program was used to implement own software controlling the operation of the signal generator.

The receiving part of the test set consisting of a vector analyzer, a computer for controlling the analyzer and recording the results and a GPS receiver was placed in a heavy-terrain vehicle, on the roof of which a receiving antenna and GPS antenna were installed at a height of about $3 \mathrm{~m}$. The measurement system implemented in this way served as a mobile sensor. The vehicle equipped with its own power generator enabled to supply all of measuring devices (Figure 4).

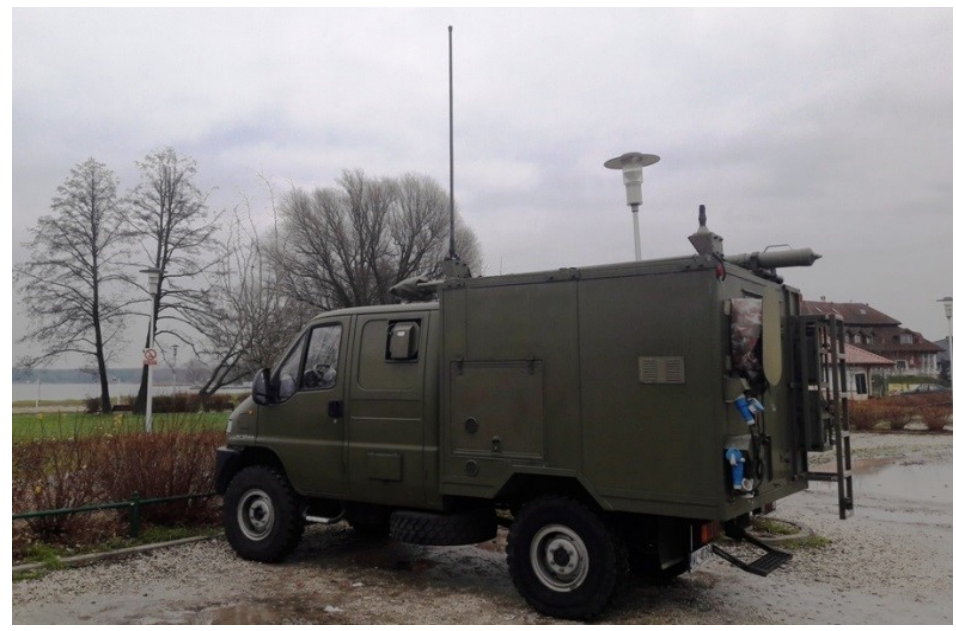

Figure 4. Mobile sensor on the heavy-terrain vehicle.

The receiving part of test system for the VHF / UHF frequency range consisted of (Figure 5):

- $\quad$ NI PXIe-5665 vector analyzer,

- $\quad 3088 \mathrm{VM}$ receiving antenna,

- $\quad$ RF-3187-AT320 receiving antenna,

- $\quad$ Garmin GPS16-HVS GPS receiver. 


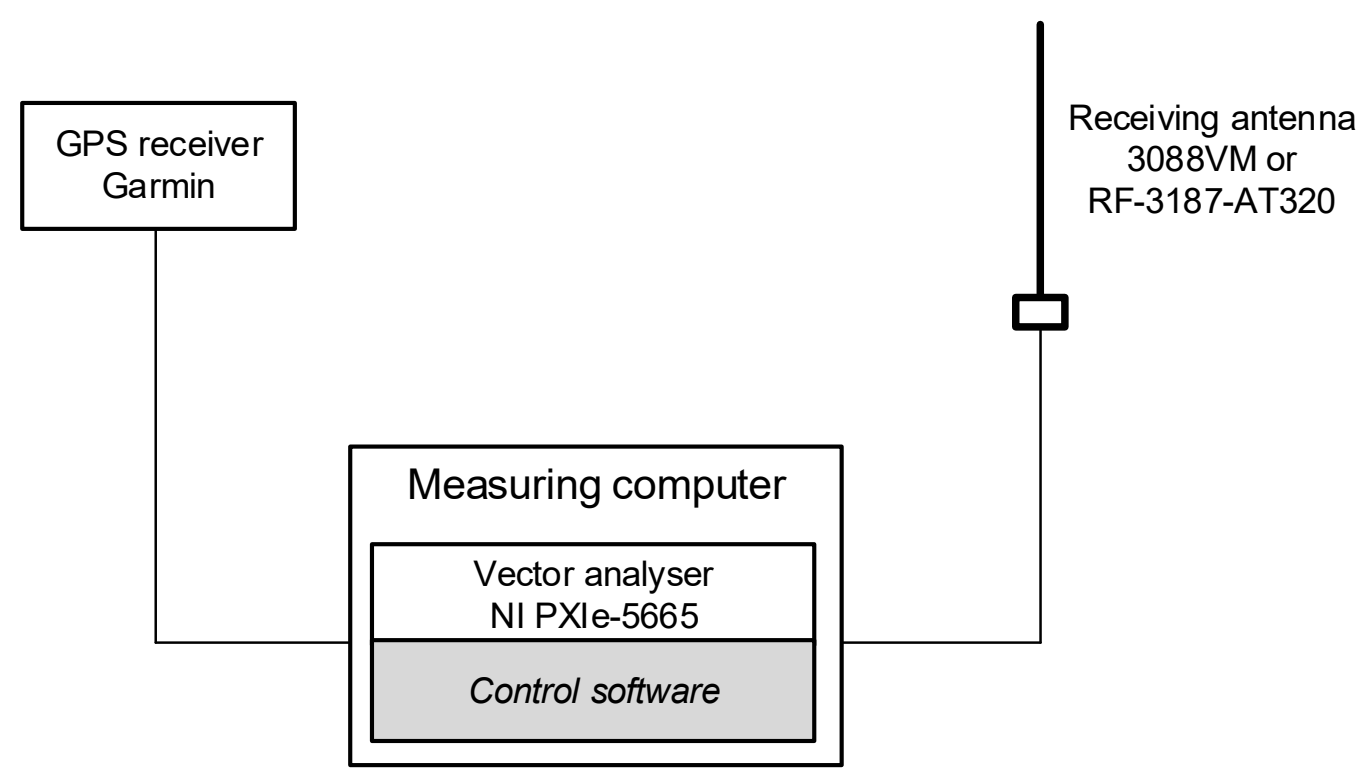

Figure 5. Receiving part of the research kit for the VHF / UHF range.

The vector signal analyzer is a device that is able to demodulate and display high frequency signals received from a vector signal generator with quadrature modulation. Demodulation process consists in extracting of the reference digital carrier signal so that it can be compared to the instantaneous phase, and then creating digital signals corresponding to temporary changes in phase and amplitude.

These changes can be visualized in the IQ plane, which allows to assess the integrity of the signal after passing through the transmission path. This makes it possible to test the quality of modulators, transmitters and other components of the transmission path that can cause transmission distortions.

The vector analyzer is a measuring device that allows to determine many parameters of modulation quality and transmission channel evaluation, such as Error Vector Magnitude (EVM), Bit Error Rate (BER) and others.

The tests were carried out in accordance with the schemes shown in Figure 3 and Figure 5.

Basic parameters of the NI PXIe-5665 vector analyzer:

- $\quad$ frequency range from $20 \mathrm{~Hz}$ to $3.6 \mathrm{GHz}$,

- $\quad$ built-in $32 \mathrm{MB}$ memory,

- $25 \mathrm{MHz}$ band,

- $\quad$ amplitude measurement accuracy: $+/-1.2 \mathrm{~dB}$.

The set of receiving antennas was identical as for the transmitting part with the antenna base installed on the roof of the vehicle.

The Garmin GPS16-HVS GPS receiver was used to synchronize measurement frequency changes and to determine the position of the mobile sensor during recording of measurement results.

The software LabVIEW was used to implement the software controlling the generator and vector analyzer. Figure 6 presents the view of the analyzer window explaining the basic measurement options used in the tests. 


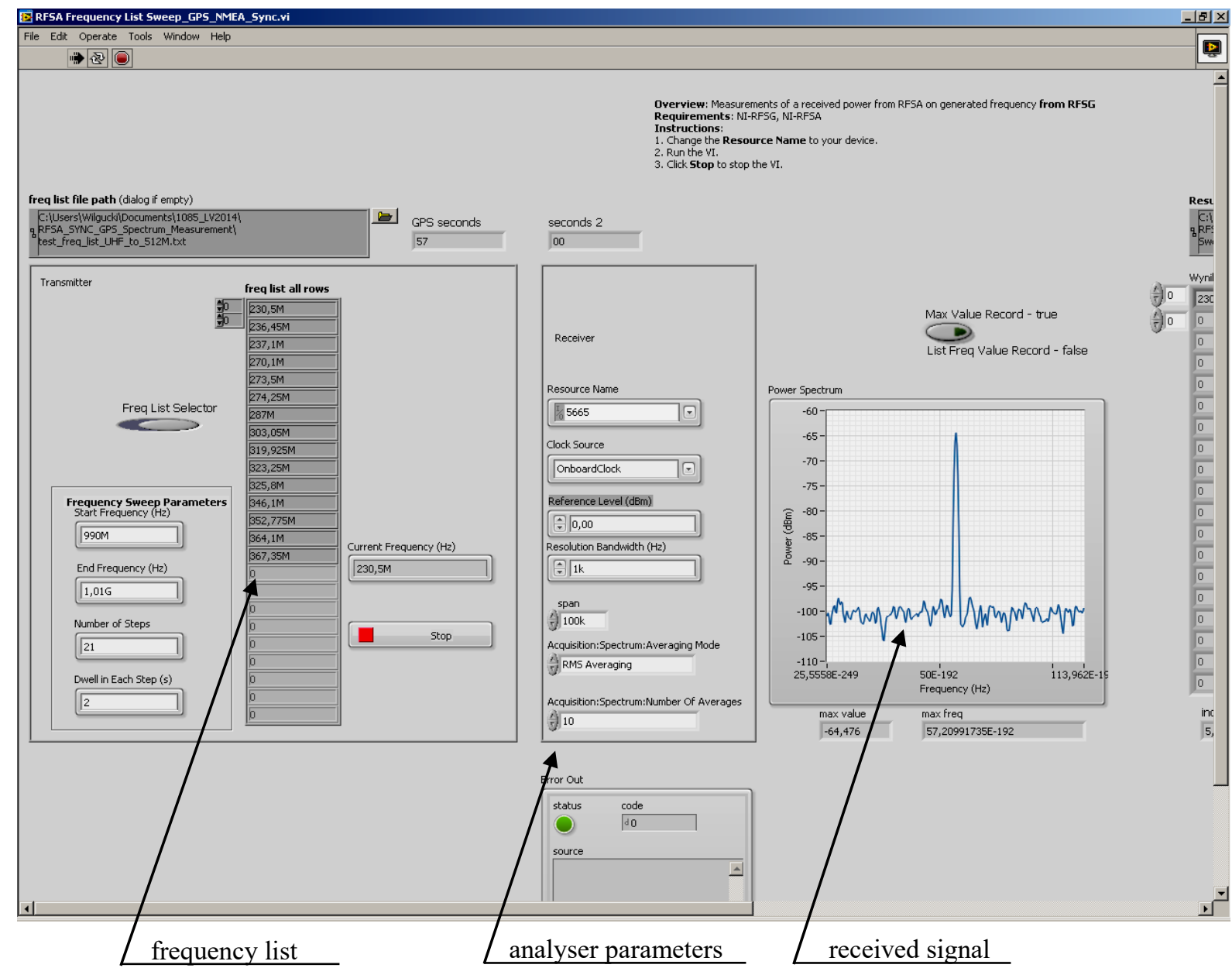

Figure 6. Basic measurement and control window.

The measurements were carried out in several concentric rings around the signal source, up to the limit of the radio signal range, and not only in one sector relative to the signal source, as was the case in some papers. Depending on the tested frequency range the radio signal range limits are marked by circles with a radius of about $3 \mathrm{~km}$ for the frequency of the upper UHF range $(0.8 \div 2 \mathrm{GHz}), 5 \div 7 \mathrm{~km}$ for the lower UHF range $(200 \div 500 \mathrm{MHz})$ and up to $15 \mathrm{~km}$ for VHF $(40 \div 100 \mathrm{MHz})$.

The measurements were carried out in a very diverse area: partly built-up area with small and medium-size buildings, partly forested area, open area (meadows), shore of the Lake and high river bank (higher the TX antenna). The type of terrain, and in particular the accessibility of access roads for the mobile sensor influenced the number of measurements performed and the way measuring points were arranged.

The heavy-terrain vehicle acting as a mobile sensor moved within the pre-selected area around the radio transmitter located on the roof of the main building of the Military Communication Institute in Zegrze (Poland). The locations of the transmitter and measuring points from P1 to P39 for tests carried out in the upper part of the UHF band are shown in Figure 7.

The following test configuration was used in the measurements: (1) frequencies from the VHF and UHF bands, (2) modulation type: CW, (3) output power: $10 \mathrm{~W},(4)$ measured parameter: average value of RSS (Received Signal Strength), (5) number of measurements taken to average: 10, (6) antenna type: omnidirectional. 


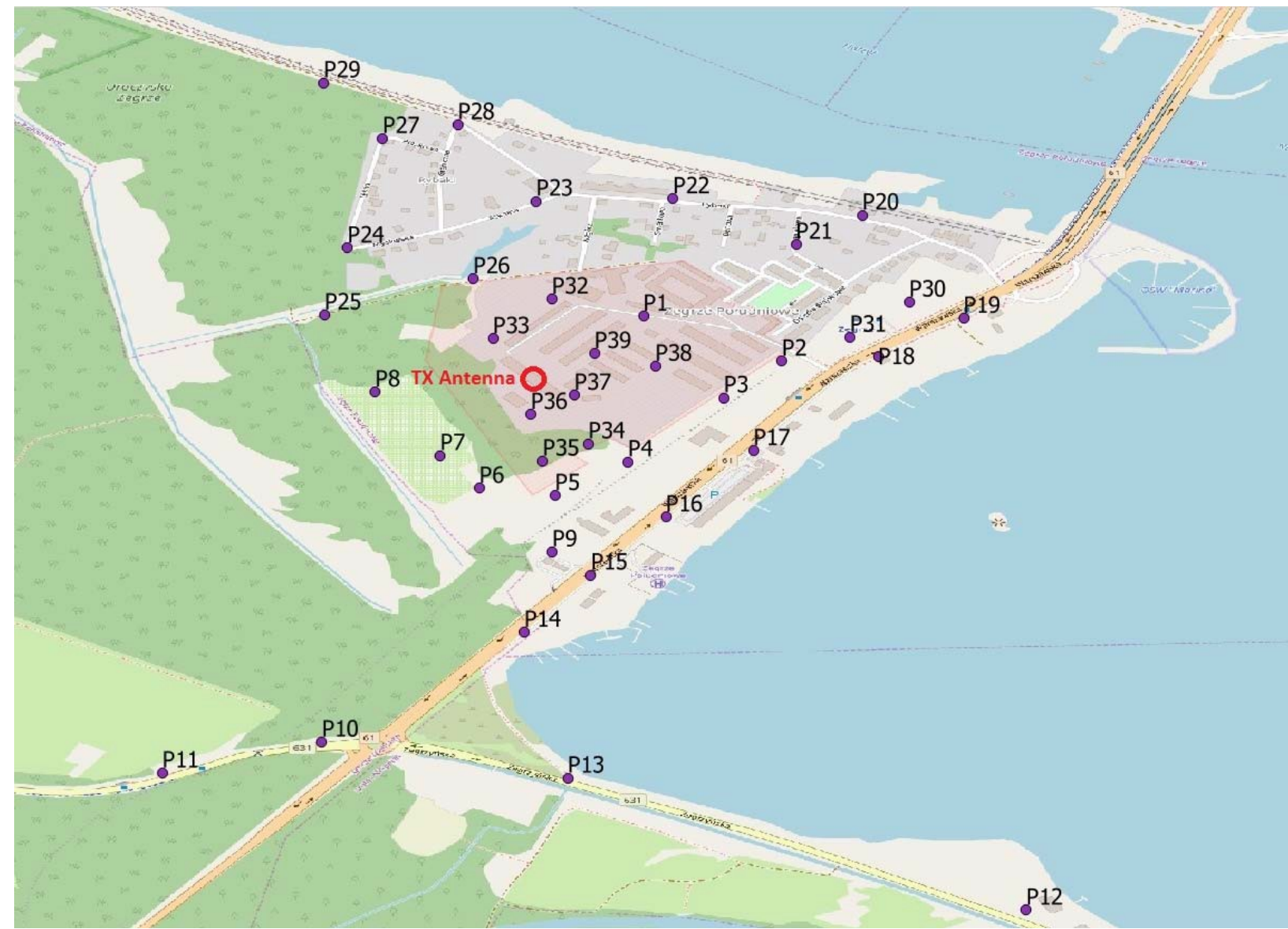

Figure 7. Distribution of measuring points and position of the transmitting antenna (TX Antenna).

The measurement results were used as input data for the interpolation process with the aim to create maps of the radio environment for the Zegrze area. The maps were evaluated for mapping accuracy of the received signal level and transmitter location. Comparison of maps obtained for various interpolation techniques allowed to select the most promising techniques for radio environment maps. The results of this work were presented at national and international conferences $[3,4,5]$ and included in publications $[6,7]$.

For the purposes of this paper Figure 8 shows examples of maps obtained for one of the sensor network deployment, and for various interpolation techniques discussed in Chapter Two: NN, IDW p1, IDW p3 and Kriging. 
a)

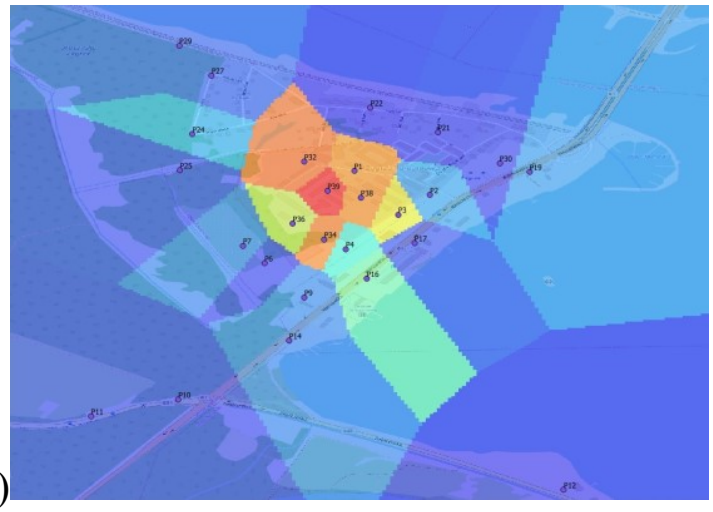

b)
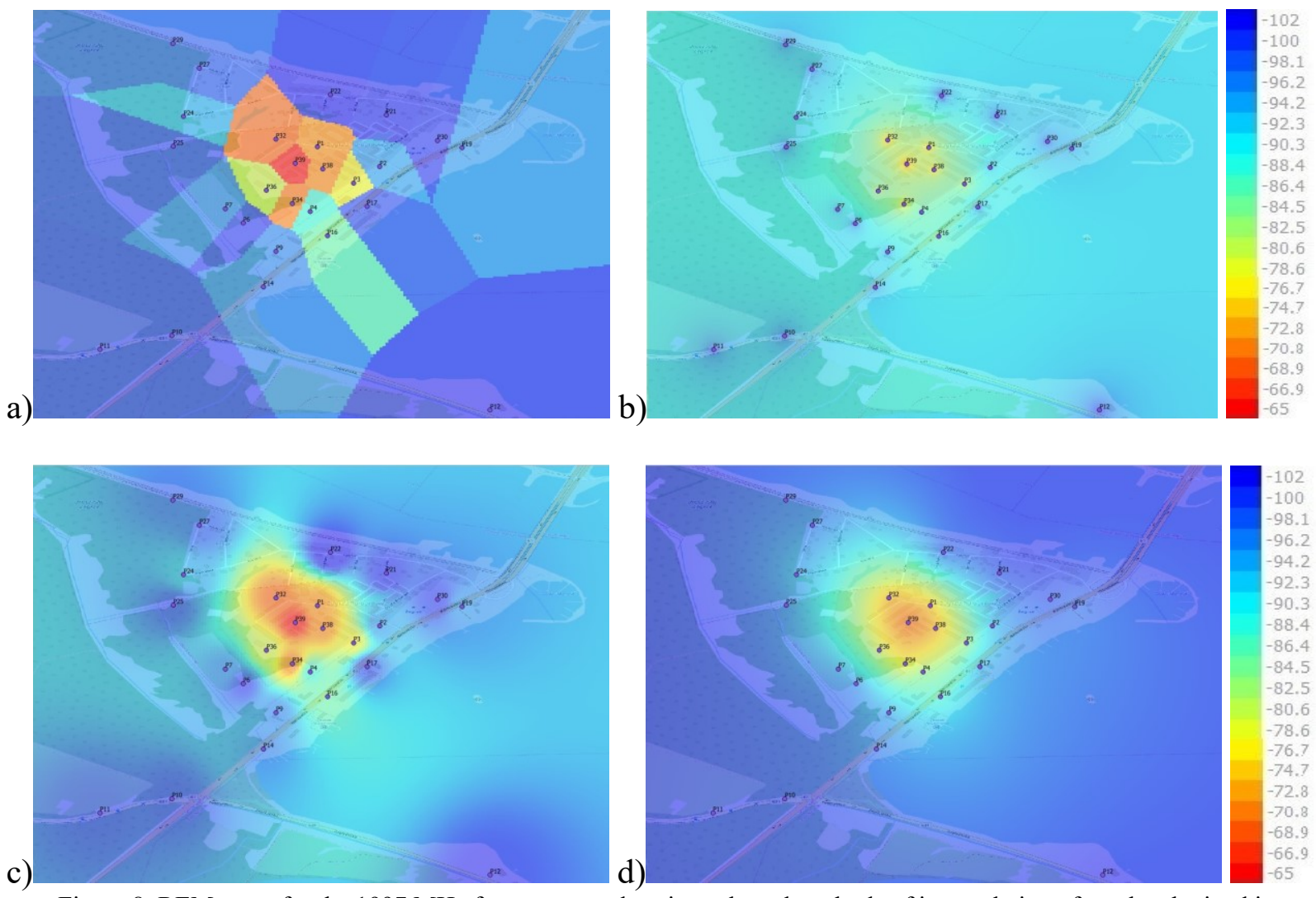

Figure 8. REM maps for the $1997 \mathrm{MHz}$ frequency made using selected methods of interpolation of results obtained in a single-transmitter test [scale in $\mathrm{dBm}$ ]: a) NN, b) IDW p1, c) IDW p3, d) Kriging.

The article also confirms that based on REM maps it is possible to indicate the location of two transmitters operating simultaneously on the same frequency. The test was performed with the use of two sources of emission operating simultaneously, in the arrangement shown in Figure 9. The distance between the antennas of the transmitters was about $200 \mathrm{~m}$. The wireless link between the transmitters was established using the Wireless Access Subsystem to control the system. The first transmitter's operation was controlled by the software while the second transmitter was controlled in the manual mode to ensure synchronous switching of the operating modes: TX 1 only, TX $1+$ TX 2 simultaneously, TX 2 only, no TX 1 and no TX 2.

The receiving part of the test set remained unchanged and was implemented in accordance with the diagram shown in Figure 5. 


\section{Sensor}

Sensor

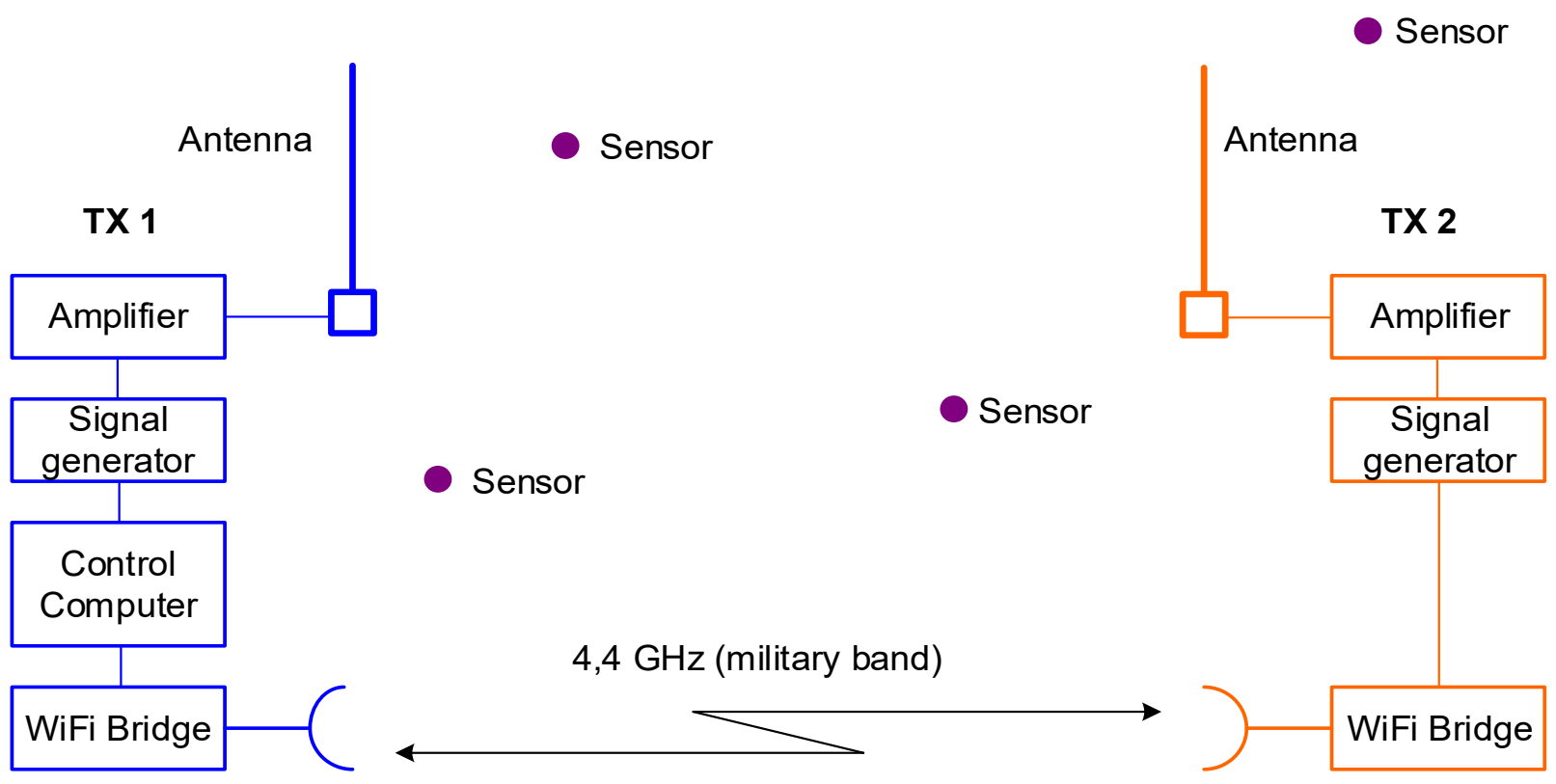

Figure 9. The transmitting part of the research kit for the VHF / UHF range with two transmitters.

Examples of maps obtained as a result of tests with two signal sources made using the IDW p3 interpolation technique and NN are shown in Figure 10. The map shows two distinct areas with the highest levels of recorded signal. Based on these maps it is possible to determine the likely locations of these sources. Survey shown that with such a short distance between two transmitters $(200 \mathrm{~m})$ their position can be determined when at least several sensors are placed between the transmitters. The lack of sensors in this important area means that it may not possible to determine whether more than one transmitters are working based on the REM maps.

a)

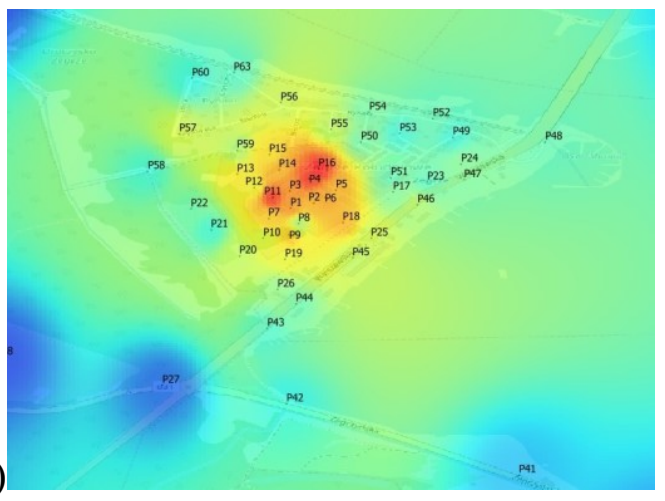

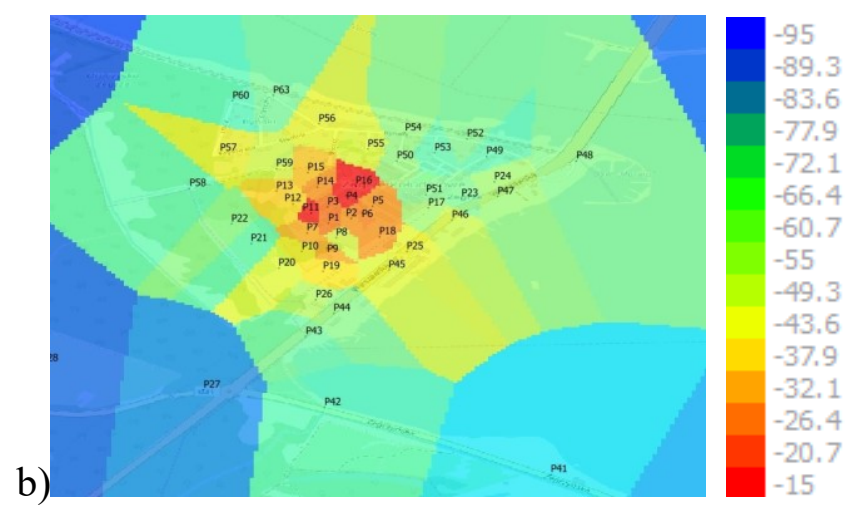

)

Figure 10. Exemplary REM maps for $236.45 \mathrm{MHz}$ frequency for selected methods of interpolation of results obtained in a test with two transmitters [scale in $\mathrm{dBm}$ ]: a) IDW p3, b) NN.

Broader discussion on the issues of determining the location of emission sources working in the VHF band based on REM maps was presented in [16]. Test for VHF band and results of analysis showed that it is possible to localize the transmitter with the accuracy from several hundred meters to several kilometers and depends on the number of sensors used to collect the results on the basis of which REM maps are constructed, as well as their location in relation to the source of emission. 


\section{CONCLUSION}

The establishment of the NATO program resulted from the need to improve the situational awareness of spectrum use and to ensure uninterrupted operation of systems based on interoperable REM databases. REM maps are considered as a promising solution that may enable the dynamic radio spectrum management, which is an essential element of radio communication based on cognitive radios.

REM maps can also be used for the needs of passive radars (PET / PCL - Passive Emitting Triangulation / Passive Coherent Location), as a preliminary recognition of the radio environment in a given location before choosing emission sources for the needs of radiolocation.

Another application is the prevention of passive radars on missions, where you can face the situation of the enemy spread their own sources (illuminators) for the needs of passive radars. These sources could be located using REM maps.

However, the main goal of the article is to present the issue of REM maps construction. It concerns the method of supplying REM database with measurement data and the method of their processing. Related to this are issues such as the placement of measurement sensors and the selection of the appropriate method of interpolation of results of measurements.

The article describes the author's measurement methodology that allows collecting of measurement data in real conditions for the purposes of creating of radio environment maps. This methodology, through the appropriate selection of measurement points taken into account in the process of creating REM maps, allowed the analysis and assessment of the impact of sensors' deployment and the selection of the most promising interpolation method. This selection is based on the analysis of the quality of created REM maps.

The measurements were carried out for two scenarios, with one and two radio signal sources. The obtained results showed that it is possible to use REM maps for the initial determination of the source of radio signal emission when there is lack of EW system support.

\section{REFERENCES}

[1] Suchański, M., Matyszkiel, R., Kaniewski, P., Kustra, M., Military Communication Institute (Poland), Gajewski, P., Łopatka, J., Military Univ. of Technology (Poland), „Dynamic spectrum management as an antiinterference method," Proceedings of SPIE Vol. 10418, SPIE, Bellingham, WA (2017), ISSN: 0277-786X, DOI: $10.1117 / 12.2269294$.

[2] Suchański, M., Gajewski, P., Łopatka, J., Kaniewski, P., Matyszkiel, R., Kustra, M., „Coordinated dynamic spectrum management in legacy military communication systems," WinnComm-Europe 2016, Wireless Innovation Forum European Conference on Communications Technologies and Software Defined Radio, Paris (2016).

[3] Suchański, M., Kaniewski, P., Romanik, J., Golan, E., Zubel, K., „Radio Environment Maps for military cognitive networks: deployment of sensors vs. map quality," International Conference on Military Communications and Information Systems (ICMCIS), Budva (2019), DOI: 10.1109/ICMCIS.2019.8842720.

[4] Suchański, M., Kaniewski, P., Romanik, J., Golan E., „Radio Environment Maps for military cognitive networks: construction techniques vs. map quality," International Conference on Military Communications and Information Systems (ICMCIS), Warsaw (2018), DOI: 10.1109/ICMCIS.2018.8398723.

[5] Suchański, M., Kaniewski, P., Romanik, J., Golan E., „Radio Environment Map to support frequency allocation in military communications systems," Baltic URSI Symposium, Poznań (2018), DOI: 10.23919/URSI.2018.8406717.

[6] Suchański, M., Kaniewski, P., Romanik, J., Golan E., Zubel, K., „Electronic Warfare systems supporting the database of the Radio Environment Maps," Proc. SPIE 11055, XII Conference on Reconnaissance and Electronic Warfare Systems, Ołtarzew (2018), DOI: 10.1117/12.2524594.

[7] Suchański, M., Kaniewski, P., Romanik, J., Golan E., Zubel, K., „Radio Environment Maps for military cognitive networks: density of sensor network vs. map quality," In: Kliks A. et al. (eds) Cognitive RadioOriented Wireless Networks. CrownCom 2019. Lecture Notes of the Institute for Computer Sciences, Social Informatics and Telecommunications Engineering, vol 291. Springer, Cham (2019), DOI: 10.1007/978-3-03025748-4_15. 
[8] Yilmaz, H. B., Tugcu, T., Alagöz, F., Bayhan, S., „Radio Environment Map as Enabler for Practical Cognitive Radio Networks," IEEE Communications Magazine, Volume: 51, Issue: 12 (2013), DOI: 10.1109/MCOM.2013.6685772.

[9] Pesko, M., Javornik, T., Košir, A., Štular, M., Mohorčič, M., „Radio environment maps: The survey of construction methods," KSII Transactions on Internet and Information Systems, vol. 8, NO. 11 (2014), DOI: 10.3837/tiis.2014.11.008.

[10] Ichikawa, K., Fujii, T., „Radio Environment Map Construction Using Hidden Markov Model in Multiple Primary User Environment," IEEE International Conference on Computing, Networking and Communications (ICCNC), Santa Clara, CA, USA (2017), DOI: 10.1109/ICCNC.2017.7876138.

[11]Frantzis, F., Chowdappa, V.-P., Botella, C., Samper, J. J., Martinez, R. J., „Radio Environment Map Estimation based on Communication Cost Modeling for Heterogeneous Networks," IEEE 85th Vehicular Technology Conference (VTC Spring), Sydney, NSW, Australia (2017), DOI: 10.1109/VTCSpring.2017.8108227.

$[12] \mathrm{Hu}$, Y., Zhang, R., „Secure Crowdsourced Radio Environment Map Construction,” IEEE 25th International Conference on Network Protocols (ICNP), Toronto, ON, Canada (2017), DOI: 10.1109/ICNP.2017.8117556.

[13] Ton, M., Phillips, C., „CRAWDAD dataset cu/wimax (v. 201206 01), traceset: samples, downloaded from https://crawdad.org/cu/wimax/20120601/samples,"(Jun 2012), DOI: 10.15783/C7BC76.

[14]Phillips, C., Ton, M., Sicker, D., Grunwald, D., „Practical Radio Environment Mapping with Geostatistics,” IEEE International Symposium on Dynamic Spectrum Access Networks, Bellevue, WA, USA (2012), DOI: 10.1109/DYSPAN.2012.6478166.

[15] Kliks, A., Kryszkiewicz, P., Cichoń, K., Umbert, A., Pérez-Romero, J., Casadevall, F., „DVB-T channels power measurements in indoor/outdoor cases," IEICE Information and Communication Technology Forum, Poznań (2014).

[16] Kaniewski, P., Golan, E., „Localization of Transmitters in VHF Band Based on the Radio Environment Maps Concept," 10th International Scientific Conference (KIT), Tatranské Zruby (2019). 